\title{
Preliminary environmental impact assessment of PFOS waste treatment in a lab-scale batch subcritical water decomposition operation
}

\author{
Jianxin Zhu $\cdot$ Shaoguo Kang $\cdot$ Yijun Xie $\cdot$ \\ Fushen Zhang
}

Received: 28 September 2012/Accepted: 20 June 2013/Published online: 13 July 2013

(C) Springer Japan 2013

\begin{abstract}
Life cycle assessment (LCA) was carried out by SimaPro 7.3 to study the environmental impact of a labscale batch subcritical water decomposition operation for a kilogram of Perfluorooctane sulfonic acid (PFOS) waste treatment in this study, a proven process for the decomposition of PFOS pollutants with high concentration. This LCA focuses on not only the main environmental factors from emissions of toxic pollutants, but also the influence from technical characteristics of the iron-induced subcritical water technology including energy and substances consumption during the subcritical water decomposition treatment process. The IMPACT 2002+ environmental model was used to evaluate the 15 midpoint and 4 endpoint environmental damages. It was found that the energy consumption to sustain the high temperature $\left(350{ }^{\circ} \mathrm{C}\right)$ and high pressure $(23 \mathrm{MPa})$ in the subcritical water process contributes $99.8 \%$ of the damages. The total negative impact of the SCWD process for $1 \mathrm{~kg}$ of PFOS waste treatment to human health, ecological quality, climate change and resources amounts to $1.11 \times 10^{-3}$, $8.43 \times 10^{-5}, 9.76 \times 10^{-4}, 9.05 \times 10^{-4} \mathrm{Pt}$, respectively. And the improvement of energy efficiency and catalytic effectiveness are two important factors to reduce the environmental impact from the SCWD process for the treatment of PFOS waste.
\end{abstract}

Designation of the 7th International Conference on Waste Management and Technology, China, 2012

J. Zhu $(\bowtie) \cdot$ S. Kang $\cdot$ Y. Xie $\cdot$ F. Zhang

Research Center for Eco-Environmental Sciences,

Chinese Academy of Sciences,

Beijing 100085, China

e-mail: zhujx@rcees.ac.cn
Keywords PFOS - Life cycle assessment . Subcritical water

\section{Introduction}

Perfluorooctane sulfonic acid (PFOS) has certain unique physiochemical properties, such as high surface-active effect, good light transparency, thermal and chemical stability, and has been worldwide used as industrial products or raw materials for surface treatment agents [1]. Although PFOS and its salts were categorized as persistent organic pollutants (POPs) at the 4th Conference of the Parties (COP4) to the Stockholm Convention in 2009, they are essential chemicals in the semiconductor industry with no cost-effective substitutes yet identified [2]. PFOS was the key ingredient of numerous stain and durable waster repellents applied to fabric, furniture and carpets [3, 4]. Together with perfluorooctanoic acid, it could be used for the production of fire-fighting and alcohol-type concentrate foams. Also, PFOS was widely used as photoacid generators and anti-reflective coatings in the semiconductor industries.

China is currently one of the few countries with increasing PFOS production and usage with an annual amount of about 200 tons, including: fabric processing 100 tons, metal plating 25 tons, semiconductor manufacturing 0.5 tons and fire-fighting foam 80 tons [5]. Recently, some research showed that PFOS contaminants could be detected in the water bodies, especially those near big cities, which mainly comes from industrial production and utilization $[6,7]$. It was also found that the PFOS concentration in the water near the semiconductor manufacturing companies was much higher than the background level, which indicates the contribution of PFOS waste 
discharged from industries is not negligible in the environment [8]. The release control of PFOS, by-product, and residuals to air, water, and land is an effective means to alleviate the nearby PFOS contamination and the environmental sound treatment and safe disposal of PFOS pollutants is critical to effectively protect human health from those toxic chemicals.

The decomposition of PFOS is difficult because the cleavage of the $\mathrm{C}-\mathrm{F}$ bonds to form fluorine ions $\left(\mathrm{F}^{-}\right)$needs high energy input. The organic $\mathrm{C}-\mathrm{F}$ bonds in PFOS yield a more stable molecular structure, such as the bond energy of $\mathrm{C}_{2} \mathrm{~F}_{5}-\mathrm{F}$ of $127 \mathrm{kcal} / \mathrm{mol}$ vs. $\mathrm{C}_{2} \mathrm{H}_{5}-\mathrm{H}$ of $101 \mathrm{kcal} / \mathrm{mol}$ [9]. The oxidative-reductive electric position of $\mathrm{F} / \mathrm{F}^{-}$is as high as $3.6 \mathrm{~V}$. Most conventional degradation technologies are ineffective for the in situ degradation of PFOS since they are inherently recalcitrant to chemical and microbiological treatment. They persist in the environment, bioaccumulate through the food web, and pose a risk of causing adverse effects to human health and the environment. Although several technologies were proven to be effective in some degree for the treatment of PFOS waste, such as persulfate photolysis, direct UV photolysis, phosphotungstic acid photocatalysis, $\mathrm{TiO}_{2}$ photocatalysis, $\mathrm{Fe}(\mathrm{II}) / \mathrm{Fe}(\mathrm{III})$ photocatalysis, UV-KI photolysis, Alkaline 2-propanol photolytic reduction and B12 mediated reduction [10], there are still many unresolved issues with those processes and the more toxic and stable by-products make them difficult to meet the requirements of PFOS treatment in an environmentally sound way.

The subcritical water decomposition reported by Hori et al. [11] shows a promising feature for the final treatment of PFOS, since PFOS could be decomposed in a subcritical water reactor without any formation of perfluorocarboxylic acids. This method was also effective in decomposing other perfluoroalkylsulforiates bearing shorter chain (C-2-C-6) perfluoroalkyl groups and was successfully applied to the decomposition of PFOS contained in an antireflective coating agent used in semiconductor manufacturing. To achieve a complete decomposition of PFOS, high temperature, long reaction time and catalyst addition was required. The high energy and material consumption will inevitably produce some negative environmental impact in line with the environment benefits from the elimination of PFOS pollutants. It is necessary to assess the environmental impacts associated with all the stages of the subcritical water decomposition of PFOS in order to improve processes and provide a sound basis for informed decisions.

Life cycle assessment (LCA) is an internationally standardized methodology for systematic and quantitative evaluation of environmental impacts of functionally equivalent products or services through all stages of their life cycles, which was widely used in waste treatment and groundwater remediation to evaluate the negative and positive impacts concerned [12, 13]. Related studies showed that LCA was an effective tool to identify environmental impacts and improve remediation technology in waste treatment and contaminated site remediation [14, 15]. This study presents a preliminary environmental impact assessment of PFOS waste treatment through LCA for a lab-scale batch subcritical water decomposing operation.

\section{Process description}

The process of PFOS decomposition in subcritical water was reported as a lab-scale batch operation [11]. The subcritical water reactor was a stainless steel high-pressure reactor equipped with a thermocouple. In a typical run, an argon-saturated aqueous mixture of PFOS waste and zerovalent iron metal powder was introduced into the reactor under an argon atmosphere, and the reactor was sealed. Then the reactor was heated to the desired reaction temperature, and the temperature was held constant for a specific time. After reaction, the reactor was cooled to room temperature and opened under an argon atmosphere, and the reaction mixture was centrifuged to separate the solution phase and the solid phase (metal powder). The solid phase could be put into a landfill and the $\mathrm{F}^{-}$containing wastewater could be treated effectively by a normal industrialized wastewater treatment process.

The input data for the material consumption in the subcritical process was reported by Hori et al. while the energy consumption for heating was calculated by energy balance in a smaller cylindrical reactor subcritical water decomposition $(S C W D)$ reactor $(\Phi 30 \times 60 \mathrm{~cm})$. When the concentration of PFOS is $93-372 \mathrm{mmol} / \mathrm{L}$, reaction in subcritical water with iron powder addition at $350{ }^{\circ} \mathrm{C}$ for $6 \mathrm{~h}$ could reduce the PFOS below the detection limit of high-performance liquid chromatography (HPLC) with conductometric detection. As for the other processes, such as mixing, separating, landfilling and wastewater treatment, the data were mainly obtained from the Ecoinvent database [16].

The fine iron metal powder could be prepared through direct atomization, electrolysis and reduction processes. Electric iron powders have good reactivity and their particle sizes could be controlled during the electrolysis process. The basic process for electric iron powder preparation includes: raw material grinding, anode preparation, electrolysis, acquisition cathode deposition of metal powder, cleaning (washing), dehydration, drying, annealing, crushing, screening and packaging. Here the process of electrolytic iron powder from a caustic soda solution was referenced since the powder could be obtained directly 
from the ore [17], in which soluble iron anodes were employed in a $50 \%$ caustic solution at $100{ }^{\circ} \mathrm{C}$. The brittle deposit prepared was suitable for crushing and very fine and dendritic powder was obtained.

\section{Life cycle assessment}

LCA (also known as life cycle analysis and cradle-to-grave analysis) is a technique to assess the impacts that a product or process makes on the environment throughout its life span. The procedures of LCA are part of the ISO 14000 environmental management standards, which is carried out in four distinct phases: (1) goal and scope definition; (2) inventory analysis; (3) impact assessment; and (4) interpretation. In this study, we performed LCA of PFOS decomposition in subcritical water using a LCA software "SimaPro 7.3" developed by PRe Consultants and a life cycle impact assessment (LCIA) method "Impact 2002+" developed by Jolliet et al. [16].

\section{Goal and scope definition}

The goal of this life cycle assessment was to assess the environmental impacts of a lab scale SCWD technology for PFOS treatment and detect high environmental impact processes for the purpose of improving its environmental performance and reducing substances consumption. The functional unit was $1 \mathrm{~kg}$ of PFOS contaminated waste from $300 \mathrm{mmol} / \mathrm{L}$ to less than the detection limit of HPLC for compiling the life cycle inventory of SCWD. The system boundary of LCA included both the first-order and secondorder impacts, covering all the direct emissions and disposal and resources required for upstream electricity generation and chemicals manufacture. Also, we define no timeframe for the LCA in order to capture both long-term and short-term impacts.

The life cycle stages for the SCWD system was grouped into four major subsystems: SUB1-Argon and fine iron powder preparation and transportation; SUB2-Heat supply in the SCWD reactor at $350{ }^{\circ} \mathrm{C}$ for $6 \mathrm{~h}$; $\mathrm{SUB} 3-\mathrm{H}_{2}$ and $\mathrm{CHF}_{3}$ gas emission; SUB4-F ${ }^{-}$containing wastewater treatment and solid residual landfill. The basic structure of the lab-scale batch SCWD for PFOS treatment is presented in Fig. 1. Here, only operation phases were considered when analyzing technologies. Impacts associated with the process of production of capital goods were excluded from this study taking into account the lack of data for the SCWD infrastructures.

There are mainly three processes in SUB1: argon preparation, iron powder preparation and their transportation. As for the argon preparation, the Ecoinvent system process of "Argon, liquid at plant/RER S" was adopted, in which the argon is produced by the fractional distillation of liquid air and hydrogen is used for the catalyst to burn oxygen impurities. Where, the "RER" means the data of the process are from Europe and the " $\mathrm{S}$ " at the end of the input name means that all processes to create the input are considered as a whole. The iron powder is prepared by an alkaline electrolysis process as referenced [17]. The process "Transport, van $<3.5 \mathrm{t} / \mathrm{RER} \mathrm{S}$ " in the Ecoinvent database was adopted for the transportation of argon and iron powder products, in which the operation of vehicle, production, maintenance and disposal of vehicles, construction and maintenance and disposal of roads were covered as unit processes.

Electricity was supposed to be used to heat the water at $350{ }^{\circ} \mathrm{C}$ for $6 \mathrm{~h}$ and the major process in SUB2 is the electricity generation and transformation. The Ecoinvent system process of "Electricity, oil, at power plant/UCTE S" was adopted in this study, in which the Union for the Coordination of the Transmission of Electricity (UCTE) specific efficiency of electricity generation and transformation was adopted. The electricity production in oil power plants with average efficiency and oil input in Europe was calculated in the process. The $\mathrm{H}_{2}$ and $\mathrm{CHF}_{3}$ emission resulting from the PFOS decomposition was covered in SUB3, which was supposed to emit into the ambient air without further treatment.

There are mainly three processes in SUB4: wastewater treatment, solid residual transportation and landfill. As for the wastewater treatment, the process of "Treatment, sewage, to wastewater treatment, class $2 / \mathrm{CH} \mathrm{S}$ " in the Ecoinvent system was adopted, which belongs to a moderately large municipal wastewater treatment plant with a design capacity of about two million gallons per day (capacity class 2). Where, "CH" means the scenario was from Switzerland. Three stage wastewater treatments (mechanical, biological, chemical) including sludge digestion (fermentation) were used in the process according to the average technology in Switzerland. As for the landfill disposal, the scenario of inert material landfill specific to the technology encountered in Switzerland for inert waste with $5 \%$ water was selected in the Ecoinvent system. In the process, no direct emissions from inert material landfill (leachate) are inventoried as deemed negligible. Module contains only exchanges to processspecific burdens (energy, land use) and infrastructure. The landfill was operated with renaturation after closure.

\section{Life cycle inventory}

In the inventory analysis phase, inputs (energy and chemicals used) and outputs (emissions to the environment) associated with the SCWD treatment processes should be analyzed in detail. And the major processes and their 
Fig. 1 Major processes and system boundary of SCWD for PFOS decomposition in a labscale batch operation
Table 1 Major processes and their contributions in the PFOS waste treatment in a lab-scale batch SCWD operation
"CH" means the scenario is from Switzerland; "RER" and "E" mean the data are from Europe; "GLO" means the scenarios belong to a global average level; "UCTE means the data are from the Union for the Co-ordination of

Transmission of Electricity; and the " $\mathrm{S}$ " at the end of the input name means that all processes to create the input are considered as a whole
Sub1

\section{Sub3}

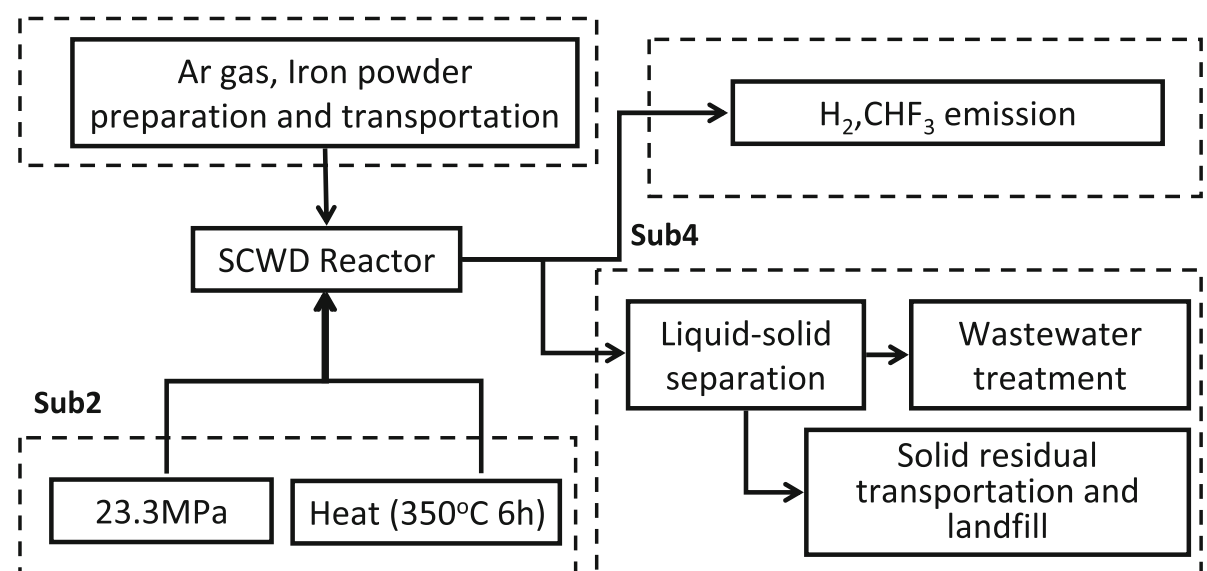

\begin{tabular}{|c|c|c|c|}
\hline Process & Project & Unit & Total \\
\hline Argon, liquid, at plant/RER $S$ & $\begin{array}{l}\text { Ecoinvent system } \\
\text { processes }\end{array}$ & $\mathrm{kg}$ & $8.92 \mathrm{E}-03$ \\
\hline $\begin{array}{l}\text { Disposal, inert waste, } 5 \% \\
\text { water, to inert material } \\
\text { landfill/CH S }\end{array}$ & $\begin{array}{l}\text { Ecoinvent system } \\
\text { processes }\end{array}$ & $\mathrm{kg}$ & $5.36 \mathrm{E}-02$ \\
\hline $\begin{array}{l}\text { Electricity, oil, at power plant/ } \\
\text { UCTE S }\end{array}$ & $\begin{array}{l}\text { Ecoinvent system } \\
\text { processes }\end{array}$ & MJ & $1.99 \mathrm{E}+01$ \\
\hline $\begin{array}{l}\text { Iron ore, } 65 \% \mathrm{Fe} \text {, at } \\
\text { beneficiation/GLO S }\end{array}$ & $\begin{array}{l}\text { Ecoinvent system } \\
\text { processes }\end{array}$ & $\mathrm{kg}$ & $8.68 \mathrm{E}-05$ \\
\hline $\begin{array}{l}\text { Sodium hydroxide } \\
\text { (concentrated) } \mathrm{E}\end{array}$ & Industry data 2.0 & $\mathrm{~kg}$ & $1.12 \mathrm{E}-04$ \\
\hline Transport, van $<3.5 \mathrm{t} / \mathrm{RER} \mathrm{S}$ & $\begin{array}{l}\text { Ecoinvent system } \\
\text { processes }\end{array}$ & tkm & $1.80 \mathrm{E}-04$ \\
\hline $\begin{array}{l}\text { Treatment, sewage, to } \\
\text { wastewater treatment, class } \\
2 / \mathrm{CH} \mathrm{S}\end{array}$ & $\begin{array}{l}\text { Ecoinvent system } \\
\text { processes }\end{array}$ & $\mathrm{m}^{3}$ & $1.00 \mathrm{E}-03$ \\
\hline $\begin{array}{l}\text { Water, decarbonised, at plant/ } \\
\text { RER S }\end{array}$ & $\begin{array}{l}\text { Ecoinvent system } \\
\text { processes }\end{array}$ & $\mathrm{kg}$ & $2.23 \mathrm{E}-04$ \\
\hline
\end{tabular}

contributions in the PFOS waste treatment are listed in Table 1. The foreground life cycle inventory (LCI) data for PFOS decomposition in the SCWD system and fine iron powder through electrolysis were compiled directly from the related papers published $[11,17]$. The background life cycle inventory data (e.g., life cycle inventory of $1 \mathrm{kWh}$ electricity, $1 \mathrm{~kg}$ of fuel oil, $1 \mathrm{~kg}$ of activated carbon) were provided by the Ecoinvent Database based on average technology data, as implemented in SimaPro 7.3. Table 2 lists the major substances used in the life cycle inventory for $1 \mathrm{~kg}$ of PFOS waste treatment in a lab-scale batch SCWD operation.

\section{Life cycle impact assessment}

The LCIA methodology adopted for this study was IMPACT 2002+, as implemented in SimaPro 7.3. This methodology proposes a feasible implementation of a combined midpoint and end-point approach by linking all types of LCI results via 15 midpoint impacts to 4 end-point damages [16]. Figure 2 shows the overall scheme of the IMPACT 2002+ framework, where the solid arrows symbolize that relevant impact pathways are known or assumed to exist and the dotted arrows indicate that these uncertain impact pathways between midpoint and endpoint levels are not modelled quantitatively. All midpoint scores are expressed in units of a reference substance and related to the four damage categories human health, ecosystem quality, climate change, and resources. These four damage categories are expressed respectively in disabilityadjusted life years (DALY), potentially disappeared fraction of species over a square meter during a year (PDF $\mathrm{m}^{2}$ year), kilogram equivalent of carbon dioxide $\left(\mathrm{kg} \mathrm{CO} \mathrm{CO}_{2-\mathrm{eq}}\right.$ ), and mega Joules (MJ).

Table 3 lists the midpoint impacts of the SCWD in a lab-scale batch operation and the contribution distribution 
Table 2 Major substances used in the life cycle inventory for $1 \mathrm{~kg}$ PFOS waste treatment in a lab-scale batch SCWD operation

\begin{tabular}{|c|c|c|c|c|c|c|c|}
\hline No & Substance & Compartment & Unit & $\begin{array}{l}\text { SUB1-Ar and iron } \\
\text { powder preparation }\end{array}$ & $\begin{array}{l}\text { SUB2-heat } \\
\text { supply }\end{array}$ & $\begin{array}{l}\text { SUB3-CHF } \\
\text { emission }\end{array}$ & $\begin{array}{l}\text { SUB4-F }{ }^{-} \text {waste } \\
\text { water treatment }\end{array}$ \\
\hline 1 & Heat, waste & Air & MJ & $6.21 \mathrm{E}-02$ & $3.11 \mathrm{E}+01$ & $x$ & $1.13 \mathrm{E}-02$ \\
\hline 2 & Carbon dioxide, fossil & Air & $\mathrm{kg}$ & $3.51 \mathrm{E}-03$ & $4.75 \mathrm{E}+00$ & $\times$ & $6.51 \mathrm{E}-04$ \\
\hline 3 & Sulfur dioxide & Air & $\mathrm{kg}$ & $1.41 \mathrm{E}-05$ & $3.64 \mathrm{E}-02$ & $\times$ & $1.89 \mathrm{E}-06$ \\
\hline 4 & Nitrogen oxides & Air & $\mathrm{kg}$ & $8.13 \mathrm{E}-06$ & $1.52 \mathrm{E}-02$ & $\times$ & $4.69 \mathrm{E}-06$ \\
\hline 5 & Argon & Air & $\mathrm{kg}$ & $\times$ & $x$ & $8.92 \mathrm{E}-03$ & $x$ \\
\hline 6 & Carbon dioxide, biogenic & Air & $\mathrm{kg}$ & $7.99 \mathrm{E}-05$ & $4.33 \mathrm{E}-03$ & $\times$ & $2.22 \mathrm{E}-04$ \\
\hline 7 & Methane, fossil & Air & $\mathrm{kg}$ & $5.83 \mathrm{E}-06$ & $2.65 \mathrm{E}-03$ & $\times$ & $1.48 \mathrm{E}-06$ \\
\hline 8 & Carbon monoxide, fossil & Air & $\mathrm{kg}$ & $3.52 \mathrm{E}-06$ & $1.69 \mathrm{E}-03$ & $\times$ & $2.03 \mathrm{E}-06$ \\
\hline 9 & $\begin{array}{l}\text { NMVOC, non-methane } \\
\text { volatile organic } \\
\text { compounds, unspecified } \\
\text { origin }\end{array}$ & Air & $\mathrm{kg}$ & $1.02 \mathrm{E}-06$ & $1.49 \mathrm{E}-03$ & $x$ & $9.23 \mathrm{E}-07$ \\
\hline 10 & Particulates, $<2.5 \mu \mathrm{m}$ & Air & $\mathrm{kg}$ & $9.22 \mathrm{E}-07$ & $6.97 \mathrm{E}-04$ & $\times$ & $3.60 \mathrm{E}-07$ \\
\hline 11 & Particulates, $>10 \mu \mathrm{m}$ & Air & $\mathrm{kg}$ & $2.08 \mathrm{E}-06$ & $5.99 \mathrm{E}-04$ & $x$ & $3.63 \mathrm{E}-07$ \\
\hline 12 & $\begin{array}{l}\text { Particulates, }>2.5 \mu \mathrm{m}, \\
\quad \text { and }<10 \mu \mathrm{m}\end{array}$ & Air & $\mathrm{kg}$ & $4.64 \mathrm{E}-07$ & $4.26 \mathrm{E}-04$ & $x$ & $2.27 \mathrm{E}-07$ \\
\hline 13 & Dinitrogen monoxide & Air & $\mathrm{kg}$ & $1.21 \mathrm{E}-07$ & $2.38 \mathrm{E}-04$ & $x$ & $1.17 \mathrm{E}-07$ \\
\hline 14 & Carbon dioxide & Air & $\mathrm{kg}$ & $1.25 \mathrm{E}-04$ & $x$ & $x$ & $x$ \\
\hline 15 & Pentane & Air & $\mathrm{kg}$ & $3.63 \mathrm{E}-08$ & $1.01 \mathrm{E}-04$ & $x$ & $1.69 \mathrm{E}-08$ \\
\hline 16 & Propane & Air & $\mathrm{kg}$ & $3.84 \mathrm{E}-08$ & $8.58 \mathrm{E}-05$ & $\times$ & $1.37 \mathrm{E}-08$ \\
\hline 17 & Oil, crude, in ground & Raw & $\mathrm{kg}$ & $3.51 \mathrm{E}-04$ & $1.39 \mathrm{E}+00$ & $x$ & $2.22 \mathrm{E}-04$ \\
\hline 18 & $\begin{array}{l}\text { Water, turbine use, } \\
\text { unspecified natural origin }\end{array}$ & Raw & $\mathrm{m}^{3}$ & $2.10 \mathrm{E}-02$ & $9.15 \mathrm{E}-01$ & $\times$ & $3.24 \mathrm{E}-03$ \\
\hline 19 & $\begin{array}{l}\text { Water, cooling, unspecified } \\
\text { natural origin } / \mathrm{m}^{3}\end{array}$ & Raw & $\mathrm{m}^{3}$ & $1.70 \mathrm{E}-04$ & $5.54 \mathrm{E}-01$ & $\times$ & $5.01 \mathrm{E}-06$ \\
\hline 20 & $\begin{array}{l}\text { Energy, potential (in } \\
\text { hydropower reservoir), } \\
\text { converted }\end{array}$ & Raw & MJ & $2.61 \mathrm{E}-03$ & $1.12 \mathrm{E}-01$ & $x$ & $4.69 \mathrm{E}-04$ \\
\hline 21 & Calcite, in ground & Raw & $\mathrm{kg}$ & $4.87 \mathrm{E}-05$ & $8.48 \mathrm{E}-02$ & $x$ & $1.47 \mathrm{E}-04$ \\
\hline 22 & Gas, natural, in ground & Raw & $\mathrm{m}^{3}$ & $2.99 \mathrm{E}-04$ & $7.40 \mathrm{E}-02$ & $\times$ & $4.23 \mathrm{E}-05$ \\
\hline 23 & Gravel, in ground & Raw & $\mathrm{kg}$ & $2.46 \mathrm{E}-04$ & $6.14 \mathrm{E}-02$ & $\times$ & $6.94 \mathrm{E}-03$ \\
\hline 24 & Coal, brown, in ground & Raw & $\mathrm{kg}$ & $1.01 \mathrm{E}-03$ & $4.17 \mathrm{E}-02$ & $\times$ & $2.91 \mathrm{E}-05$ \\
\hline 25 & $\begin{array}{l}\text { Energy, gross calorific } \\
\text { value, in biomass }\end{array}$ & Raw & MJ & $7.59 \mathrm{E}-04$ & $3.57 \mathrm{E}-02$ & $\times$ & $5.22 \mathrm{E}-05$ \\
\hline 26 & $\begin{array}{l}\text { Coal, hard, unspecified, in } \\
\text { ground }\end{array}$ & Raw & $\mathrm{kg}$ & $5.65 \mathrm{E}-04$ & $3.32 \mathrm{E}-02$ & $x$ & $5.22 \mathrm{E}-05$ \\
\hline 27 & $\begin{array}{l}\text { Energy, kinetic (in wind), } \\
\text { converted }\end{array}$ & Raw & MJ & $4.17 \mathrm{E}-04$ & $1.71 \mathrm{E}-02$ & $\times$ & $1.19 \mathrm{E}-05$ \\
\hline 28 & $\begin{array}{l}\text { Iron, } 46 \% \text { in ore, } 25 \% \text { in } \\
\text { crude ore, in ground }\end{array}$ & Raw & $\mathrm{kg}$ & $8.10 \mathrm{E}-05$ & $1.30 \mathrm{E}-02$ & $x$ & $3.28 \mathrm{E}-05$ \\
\hline 29 & $\begin{array}{l}\text { Barite, } 15 \% \text { in crude ore, } \\
\text { in ground }\end{array}$ & Raw & $\mathrm{kg}$ & $1.79 \mathrm{E}-06$ & $7.07 \mathrm{E}-03$ & $\times$ & $6.58 \mathrm{E}-07$ \\
\hline 30 & Water, river & Raw & $\mathrm{m}^{3}$ & $1.89 \mathrm{E}-05$ & $4.96 \mathrm{E}-03$ & $x$ & $3.70 \mathrm{E}-06$ \\
\hline 31 & Occupation, industrial area & Raw & $\mathrm{m}^{2} \mathrm{a}$ & $2.82 \mathrm{E}-06$ & $4.61 \mathrm{E}-03$ & $x$ & $5.77 \mathrm{E}-07$ \\
\hline 32 & Carbon dioxide, in air & Raw & $\mathrm{kg}$ & $8.48 \mathrm{E}-05$ & $3.88 \mathrm{E}-03$ & $\times$ & $5.32 \mathrm{E}-06$ \\
\hline 33 & $\begin{array}{l}\text { Water, unspecified natural } \\
\text { origin } / \mathrm{m}^{3}\end{array}$ & Raw & $\mathrm{m}^{3}$ & $1.83 \mathrm{E}-06$ & $3.60 \mathrm{E}-03$ & $x$ & $1.07 \mathrm{E}-05$ \\
\hline 34 & Clay, unspecified, in ground & Raw & $\mathrm{kg}$ & $4.91 \mathrm{E}-06$ & $3.17 \mathrm{E}-03$ & $\times$ & $5.23 \mathrm{E}-05$ \\
\hline 35 & $\begin{array}{l}\text { Occupation, forest, } \\
\text { intensive, normal }\end{array}$ & Raw & $\mathrm{m}^{2} \mathrm{a}$ & $3.70 \mathrm{E}-05$ & $2.48 \mathrm{E}-03$ & $\times$ & $5.28 \mathrm{E}-06$ \\
\hline 36 & Volume occupied, reservoir & Raw & $\mathrm{m}^{3} \mathrm{y}$ & $4.82 \mathrm{E}-05$ & $2.00 \mathrm{E}-03$ & $\times$ & $8.81 \mathrm{E}-06$ \\
\hline
\end{tabular}


Table 2 continued

\begin{tabular}{|c|c|c|c|c|c|c|c|}
\hline No & Substance & Compartment & Unit & $\begin{array}{l}\text { SUB1-Ar and iron } \\
\text { powder preparation }\end{array}$ & $\begin{array}{l}\text { SUB2-heat } \\
\text { supply }\end{array}$ & $\begin{array}{l}\mathrm{SUB}_{3}-\mathrm{CHF}_{3} \\
\text { emission }\end{array}$ & $\begin{array}{l}\text { SUB4-F- waste } \\
\text { water treatment }\end{array}$ \\
\hline 37 & Transformation, from forest & Raw & $\mathrm{m}^{2}$ & $4.53 \mathrm{E}-07$ & $1.79 \mathrm{E}-03$ & $x$ & $1.66 \mathrm{E}-07$ \\
\hline 38 & $\begin{array}{l}\text { Transformation, to mineral } \\
\text { extraction site }\end{array}$ & Raw & $\mathrm{m}^{2}$ & $4.75 \mathrm{E}-07$ & $1.71 \mathrm{E}-03$ & $\times$ & $3.55 \mathrm{E}-07$ \\
\hline 39 & Water, salt, sole & Raw & $\mathrm{m}^{3}$ & $2.46 \mathrm{E}-07$ & $1.10 \mathrm{E}-03$ & $\times$ & $1.21 \mathrm{E}-07$ \\
\hline 40 & $\begin{array}{l}\text { Occupation, traffic area, } \\
\text { road network }\end{array}$ & Raw & $\mathrm{m}^{2} \mathrm{a}$ & $7.87 \mathrm{E}-06$ & $1.01 \mathrm{E}-03$ & $x$ & $2.50 \mathrm{E}-05$ \\
\hline 41 & Energy, from gas, natural & Raw & MJ & $1.00 \mathrm{E}-03$ & $x$ & $x$ & $x$ \\
\hline 42 & Sodium chloride, in ground & Raw & $\mathrm{kg}$ & $1.16 \mathrm{E}-04$ & $8.64 \mathrm{E}-04$ & $x$ & $1.03 \mathrm{E}-05$ \\
\hline 43 & $\begin{array}{l}\text { Water, cooling, unspecified } \\
\text { natural origin } / \mathrm{kg}\end{array}$ & Raw & $\mathrm{kg}$ & $7.37 \mathrm{E}-04$ & $\times$ & $x$ & $x$ \\
\hline 44 & Energy, from uranium & Raw & MJ & $6.12 \mathrm{E}-04$ & $\times$ & $\times$ & $\times$ \\
\hline 45 & Clay, bentonite, in ground & Raw & $\mathrm{kg}$ & $4.55 \mathrm{E}-07$ & $5.55 \mathrm{E}-04$ & $x$ & $4.55 \mathrm{E}-07$ \\
\hline 46 & Water, salt, ocean & Raw & $\mathrm{m}^{3}$ & $2.90 \mathrm{E}-06$ & $5.44 \mathrm{E}-04$ & $x$ & $1.95 \mathrm{E}-07$ \\
\hline 47 & $\begin{array}{l}\text { Transformation, from sea } \\
\text { and ocean }\end{array}$ & Raw & $\mathrm{m}^{2}$ & $1.58 \mathrm{E}-07$ & $5.21 \mathrm{E}-04$ & $x$ & $3.55 \mathrm{E}-08$ \\
\hline 48 & $\begin{array}{l}\text { Transformation, to dump } \\
\text { site, benthos }\end{array}$ & Raw & $\mathrm{m}^{2}$ & $1.57 \mathrm{E}-07$ & $5.21 \mathrm{E}-04$ & $x$ & $3.54 \mathrm{E}-08$ \\
\hline 49 & $\begin{array}{l}\text { Occupation, dump site, } \\
\text { benthos }\end{array}$ & Raw & $\mathrm{m}^{2} \mathrm{a}$ & $1.57 \mathrm{E}-07$ & $5.21 \mathrm{E}-04$ & $x$ & $3.54 \mathrm{E}-08$ \\
\hline 50 & $\begin{array}{l}\text { Occupation, water bodies, } \\
\text { artificial }\end{array}$ & Raw & $\mathrm{m}^{2} \mathrm{a}$ & $5.01 \mathrm{E}-06$ & $4.28 \mathrm{E}-04$ & $x$ & $7.72 \mathrm{E}-07$ \\
\hline 51 & Energy, from coal & Raw & MJ & $4.33 \mathrm{E}-04$ & $\times$ & $x$ & $x$ \\
\hline 52 & $\begin{array}{l}\text { Occupation, water courses, } \\
\text { artificial }\end{array}$ & Raw & $\mathrm{m}^{2} \mathrm{a}$ & $2.27 \mathrm{E}-06$ & $4.15 \mathrm{E}-04$ & $x$ & $4.67 \mathrm{E}-07$ \\
\hline 53 & $\begin{array}{l}\text { Occupation, mineral } \\
\text { extraction site }\end{array}$ & Raw & $\mathrm{m}^{2} \mathrm{a}$ & $2.41 \mathrm{E}-06$ & $4.04 \mathrm{E}-04$ & $x$ & $2.12 \mathrm{E}-06$ \\
\hline 54 & $\begin{array}{l}\text { Water, process, unspecified } \\
\text { natural origin } / \mathrm{kg}\end{array}$ & Raw & $\mathrm{kg}$ & $3.33 \mathrm{E}-04$ & $\times$ & $x$ & $x$ \\
\hline 55 & $\begin{array}{l}\text { Gas, mine, off-gas, process, } \\
\text { coal mining } / \mathrm{m}^{3}\end{array}$ & Raw & $\mathrm{m}^{3}$ & $5.48 \mathrm{E}-06$ & $3.24 \mathrm{E}-04$ & $x$ & $5.31 \mathrm{E}-07$ \\
\hline 56 & Water, well, in ground & Raw & $\mathrm{m}^{3}$ & $4.20 \mathrm{E}-06$ & $2.79 \mathrm{E}-04$ & $\times$ & $1.60 \mathrm{E}-06$ \\
\hline 57 & Occupation, dump site & Raw & $\mathrm{m}^{2} \mathrm{a}$ & $3.38 \mathrm{E}-06$ & $2.28 \mathrm{E}-04$ & $x$ & $2.47 \mathrm{E}-05$ \\
\hline 58 & Energy, from oil & Raw & MJ & $2.54 \mathrm{E}-04$ & $\times$ & $x$ & $x$ \\
\hline 59 & Energy, solar, converted & Raw & MJ & $6.00 \mathrm{E}-06$ & $2.47 \mathrm{E}-04$ & $x$ & $4.34 \mathrm{E}-07$ \\
\hline 60 & $\begin{array}{l}\text { Nickel, } 1.98 \% \text { in silicates, } \\
1.04 \% \text { in crude ore, in } \\
\text { ground }\end{array}$ & Raw & $\mathrm{kg}$ & $6.92 \mathrm{E}-07$ & $2.13 \mathrm{E}-04$ & $x$ & $2.85 \mathrm{E}-06$ \\
\hline 61 & $\begin{array}{l}\text { Magnesite, } 60 \% \text { in crude } \\
\text { ore, in ground }\end{array}$ & Raw & $\mathrm{kg}$ & $1.82 \mathrm{E}-07$ & $1.72 \mathrm{E}-04$ & $x$ & $5.22 \mathrm{E}-07$ \\
\hline 62 & $\begin{array}{l}\text { Occupation, construction } \\
\text { site }\end{array}$ & Raw & $\mathrm{m}^{2} \mathrm{a}$ & $1.09 \mathrm{E}-07$ & $1.63 \mathrm{E}-04$ & $x$ & $2.60 \mathrm{E}-06$ \\
\hline 63 & $\begin{array}{l}\text { Occupation, industrial area, } \\
\text { built up }\end{array}$ & Raw & $\mathrm{m}^{2} \mathrm{a}$ & $2.38 \mathrm{E}-07$ & $1.52 \mathrm{E}-04$ & $x$ & $2.25 \mathrm{E}-06$ \\
\hline 64 & Energy, from hydro power & Raw & MJ & $1.12 \mathrm{E}-04$ & $x$ & $\times$ & $x$ \\
\hline 65 & Fluorspar, $92 \%$, in ground & Raw & $\mathrm{kg}$ & $8.91 \mathrm{E}-08$ & $1.11 \mathrm{E}-04$ & $x$ & $3.34 \mathrm{E}-08$ \\
\hline 66 & $\begin{array}{l}\text { Energy, gross calorific } \\
\text { value, in biomass, } \\
\text { primary forest }\end{array}$ & Raw & MJ & $3.74 \mathrm{E}-08$ & $9.14 \mathrm{E}-05$ & $x$ & $2.01 \mathrm{E}-08$ \\
\hline 67 & $\begin{array}{l}\text { Occupation, industrial area, } \\
\text { vegetation }\end{array}$ & Raw & $\mathrm{m}^{2} \mathrm{a}$ & $1.62 \mathrm{E}-07$ & $8.42 \mathrm{E}-05$ & $x$ & $2.20 \mathrm{E}-06$ \\
\hline
\end{tabular}


Table 2 continued

\begin{tabular}{|c|c|c|c|c|c|c|c|}
\hline No & Substance & Compartment & Unit & $\begin{array}{l}\text { SUB1-Ar and iron } \\
\text { powder preparation }\end{array}$ & $\begin{array}{l}\text { SUB2-heat } \\
\text { supply }\end{array}$ & $\begin{array}{l}\text { SUB3-CHF } \\
\text { emission }\end{array}$ & $\begin{array}{l}\text { SUB4-F- waste } \\
\text { water treatment }\end{array}$ \\
\hline 68 & $\begin{array}{l}\text { Transformation, to } \\
\text { heterogeneous, } \\
\text { agricultural }\end{array}$ & Raw & $\mathrm{m}^{2}$ & $2.11 \mathrm{E}-08$ & $8.34 \mathrm{E}-05$ & $x$ & $9.65 \mathrm{E}-09$ \\
\hline 69 & Heat, waste & Soil & MJ & $1.18 \mathrm{E}-04$ & $1.10 \mathrm{E}-02$ & $x$ & $6.80 \mathrm{E}-05$ \\
\hline 70 & Oils, unspecified & Soil & $\mathrm{kg}$ & $1.34 \mathrm{E}-06$ & $6.40 \mathrm{E}-03$ & $x$ & $3.98 \mathrm{E}-07$ \\
\hline 71 & Calcium & Soil & $\mathrm{kg}$ & $6.44 \mathrm{E}-08$ & $1.66 \mathrm{E}-04$ & $x$ & $1.94 \mathrm{E}-06$ \\
\hline 72 & Chloride & Soil & $\mathrm{kg}$ & $1.77 \mathrm{E}-06$ & $1.57 \mathrm{E}-04$ & $x$ & $2.75 \mathrm{E}-07$ \\
\hline 73 & Carbon & Soil & $\mathrm{kg}$ & $3.19 \mathrm{E}-08$ & $1.24 \mathrm{E}-04$ & $x$ & $6.70 \mathrm{E}-06$ \\
\hline 74 & Iron & Soil & $\mathrm{kg}$ & $3.99 \mathrm{E}-08$ & $8.93 \mathrm{E}-05$ & $x$ & $5.15 \mathrm{E}-06$ \\
\hline 75 & Sodium & Soil & $\mathrm{kg}$ & $2.49 \mathrm{E}-08$ & $8.25 \mathrm{E}-05$ & $x$ & $8.50 \mathrm{E}-09$ \\
\hline 76 & Heat, waste & Water & MJ & $5.14 \mathrm{E}-03$ & $1.30 \mathrm{E}+01$ & $x$ & $1.57 \mathrm{E}-03$ \\
\hline 77 & Chloride & Water & $\mathrm{kg}$ & $2.61 \mathrm{E}-05$ & $4.66 \mathrm{E}-02$ & $x$ & $4.74 \mathrm{E}-05$ \\
\hline 78 & Sodium, ion & Water & $\mathrm{kg}$ & $3.45 \mathrm{E}-05$ & $2.82 \mathrm{E}-02$ & $x$ & $7.53 \mathrm{E}-06$ \\
\hline 79 & Sulfate & Water & $\mathrm{kg}$ & $2.12 \mathrm{E}-04$ & $2.01 \mathrm{E}-02$ & $x$ & $1.60 \mathrm{E}-04$ \\
\hline 80 & $\begin{array}{l}\text { COD, chemical oxygen } \\
\text { demand }\end{array}$ & Water & $\mathrm{kg}$ & $4.35 \mathrm{E}-06$ & $1.96 \mathrm{E}-02$ & $x$ & $3.27 \mathrm{E}-05$ \\
\hline 81 & $\begin{array}{l}\text { BOD5, biological oxygen } \\
\text { demand }\end{array}$ & Water & $\mathrm{kg}$ & $4.16 \mathrm{E}-06$ & $1.94 \mathrm{E}-02$ & $x$ & $1.16 \mathrm{E}-05$ \\
\hline 82 & Calcium, ion & Water & $\mathrm{kg}$ & $5.31 \mathrm{E}-05$ & $6.39 \mathrm{E}-03$ & $x$ & $8.54 \mathrm{E}-05$ \\
\hline 83 & Oils, unspecified & Water & $\mathrm{kg}$ & $1.28 \mathrm{E}-06$ & $6.06 \mathrm{E}-03$ & $x$ & $4.29 \mathrm{E}-07$ \\
\hline 84 & TOC, total organic carbon & Water & $\mathrm{kg}$ & $1.35 \mathrm{E}-06$ & $5.98 \mathrm{E}-03$ & $x$ & $8.18 \mathrm{E}-06$ \\
\hline 85 & $\begin{array}{l}\text { DOC, dissolved organic } \\
\text { carbon }\end{array}$ & Water & $\mathrm{kg}$ & $1.34 \mathrm{E}-06$ & $5.92 \mathrm{E}-03$ & $x$ & $8.42 \mathrm{E}-06$ \\
\hline 86 & Silicon & Water & $\mathrm{kg}$ & $3.91 \mathrm{E}-05$ & $2.08 \mathrm{E}-03$ & $x$ & $6.01 \mathrm{E}-06$ \\
\hline 87 & Magnesium & Water & $\mathrm{kg}$ & $2.57 \mathrm{E}-05$ & $1.65 \mathrm{E}-03$ & $x$ & $6.89 \mathrm{E}-06$ \\
\hline 88 & $\begin{array}{l}\text { Suspended solids, } \\
\text { unspecified }\end{array}$ & Water & $\mathrm{kg}$ & $8.27 \mathrm{E}-07$ & $1.32 \mathrm{E}-03$ & $x$ & $1.12 \mathrm{E}-07$ \\
\hline 89 & Potassium, ion & Water & $\mathrm{kg}$ & $1.49 \mathrm{E}-05$ & $1.08 \mathrm{E}-03$ & $x$ & $1.25 \mathrm{E}-06$ \\
\hline 90 & Nitrogen & Water & $\mathrm{kg}$ & $6.85 \mathrm{E}-08$ & $4.17 \mathrm{E}-04$ & $x$ & $4.91 \mathrm{E}-07$ \\
\hline 91 & Phosphate & Water & $\mathrm{kg}$ & $8.45 \mathrm{E}-06$ & $3.93 \mathrm{E}-04$ & $x$ & $3.28 \mathrm{E}-06$ \\
\hline 92 & Iron, ion & Water & $\mathrm{kg}$ & $7.34 \mathrm{E}-06$ & $3.77 \mathrm{E}-04$ & $x$ & $8.05 \mathrm{E}-06$ \\
\hline 93 & $\begin{array}{l}\text { Carboxylic acids, } \\
\text { unspecified }\end{array}$ & Water & $\mathrm{kg}$ & $8.60 \mathrm{E}-08$ & $3.85 \mathrm{E}-04$ & $x$ & $4.31 \mathrm{E}-08$ \\
\hline 94 & Aluminium & Water & $\mathrm{kg}$ & $4.60 \mathrm{E}-06$ & $3.44 \mathrm{E}-04$ & $x$ & $3.64 \mathrm{E}-06$ \\
\hline 95 & Barite & Water & $\mathrm{kg}$ & $9.81 \mathrm{E}-08$ & $3.24 \mathrm{E}-04$ & $x$ & $2.21 \mathrm{E}-08$ \\
\hline 96 & Strontium & Water & $\mathrm{kg}$ & $9.10 \mathrm{E}-07$ & $2.00 \mathrm{E}-04$ & $x$ & $5.96 \mathrm{E}-08$ \\
\hline 97 & Nitrate & Water & $\mathrm{kg}$ & $2.53 \mathrm{E}-06$ & $1.21 \mathrm{E}-04$ & $x$ & $4.85 \mathrm{E}-05$ \\
\hline 98 & Solids, inorganic & Water & $\mathrm{kg}$ & $3.57 \mathrm{E}-06$ & $1.60 \mathrm{E}-04$ & $x$ & $1.27 \mathrm{E}-07$ \\
\hline 99 & Manganese & Water & $\mathrm{kg}$ & $1.99 \mathrm{E}-06$ & $9.85 \mathrm{E}-05$ & $x$ & $1.93 \mathrm{E}-07$ \\
\hline 100 & Sulfite & Water & $\mathrm{kg}$ & $1.51 \mathrm{E}-08$ & $9.12 \mathrm{E}-05$ & $x$ & $1.49 \mathrm{E}-10$ \\
\hline
\end{tabular}

of SUB1, SUB2, SUB3 and SUB4 for $1 \mathrm{~kg}$ PFOS waste treatment. It could be found that more than $97 \%$ of the midpoint environmental impacts were contributed by SUB2-Heat supply to sustain the high temperature of $350{ }^{\circ} \mathrm{C}$ and the high pressure of $23 \mathrm{MPa}$ in the SCWD reactor. The other subsystems SUB1-Ar and iron powder preparation and SUB4- $\mathrm{F}^{-}$wastewater treatment only contribute less than $3 \%$ of the midpoint impacts. As for
SUB3- $\mathrm{CHF}_{3}$ and $\mathrm{H}_{2}$ emission, it contributes only $0.3 \%$ on the global warming impact from its greenhouse gas emission. The major sources of midpoint impacts from SUB2 were specially analyzed in the following.

Human toxicity (carcinogenic and non-carcinogenic effects) represents all effects on human health in IMPACT2002+, in which the cumulative toxicological risk and potential impacts associated with a specified mass 
Fig. 2 Overall scheme of the IMPACT 2002+ framework (Full arrows symbolize that a relevant impact pathway is known and quantitatively modeled. Dotted arrows represent impact pathways that are assumed to exist, but that are not modeled.)

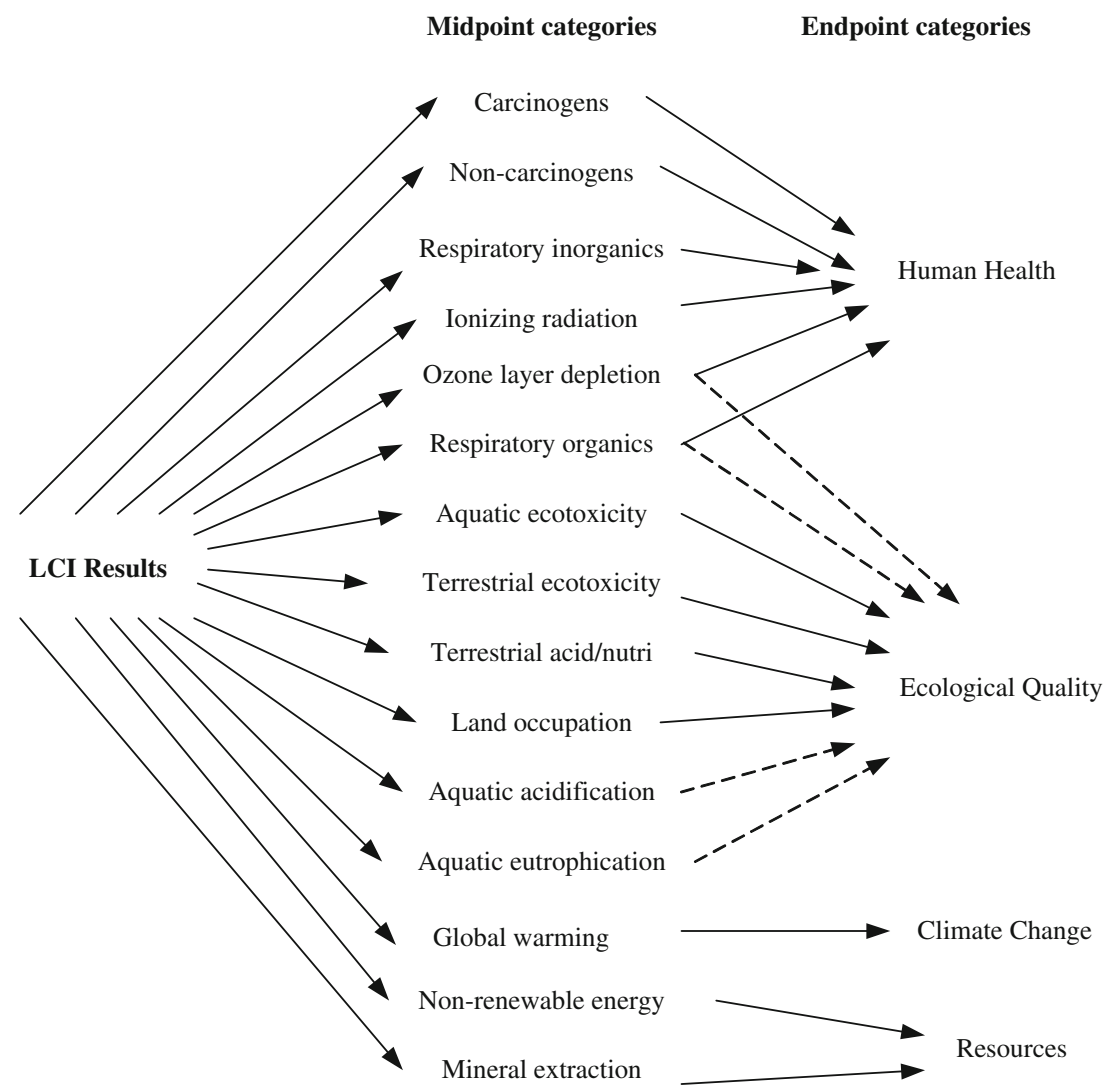

$(\mathrm{kg})$ of a chemical emitted into the environment are determined by the chemical toxic model [18]. The reference substance is chloroethylene $\left(\mathrm{C}_{2} \mathrm{H}_{3} \mathrm{Cl}\right)$ emitted into air for the midpoint characterization factors ( $\mathrm{CFs}$ ) of human toxicity and the $\mathrm{CFs}$ are expressed in kilogram equivalent of chloroethylene into air $\left(\mathrm{kg} \mathrm{C}_{2} \mathrm{H}_{3} \mathrm{Cl}_{\text {-eq }}\right)$. The electric power consumption is about $0.4542 \mathrm{kWh}$ to heat $1 \mathrm{~kg}$ PFOS containing waste from $20{ }^{\circ} \mathrm{C}$ at 1 atm pressure to $350{ }^{\circ} \mathrm{C}$ at $23.3 \mathrm{MPa}$ in SUB2. When a smaller cylindrical reactor SCWD reactor $(\Phi 30 \times 60 \mathrm{~cm})$ is supposed to be used in the operation, the heat consumption to hold the high temperature for $6 \mathrm{~h}$ in a batch operation is about $5.5135 \mathrm{kWh}$. The carcinogenic effects of aromatic hydrocarbons discharged to water and emitted into air, dioxins and arsenic emitted into air compartment from fossil fuel burning for electricity generation are as high as $4.80 \times 10^{-3}, 4.42 \times 10^{-3}, \quad 1.17 \times 10^{-3}$ and $5.70 \times$ $10^{-4} \mathrm{~kg} \mathrm{C}_{2} \mathrm{H}_{3} \mathrm{Cl}_{\text {-eq }}$, as shown in Table 4, respectively. They account for more than $90.3 \%$ of the total carcinogenic damage effects. The major non-carcinogenic substances considered in SUB2 are dioxins and arsenic emitted into the air, barium discharged to water, and arsenic released to soil, their non-carcinogenic contributions are about $5.92 \times 10^{-3}, \quad 5.72 \times 10^{-3}, \quad 2.56 \times 10^{-3}$ and $1.68 \times 10^{-3} \mathrm{~kg} \mathrm{C}_{2} \mathrm{H}_{3} \mathrm{Cl}_{-\mathrm{eq}}$, as shown in Table 5, respectively. They take more than $76.6 \%$ of the whole non-carcinogenic damage effects.

Respiratory effects caused by inorganics were expressed in kilogram equivalents of $\mathrm{PM}_{2.5}$ into air $(\mathrm{kg}$ PM2.5 into air $_{\text {-eq }}$ ) and obtained by dividing the damage factor of the considered substance by the damage factor of the reference substance [19]. The air emissions of sulfur dioxide, nitrogen oxides and $\mathrm{PM}_{2.5}$ from the electricity generation in SUB2 are about $2.84 \times 10^{-3}, \quad 1.93 \times 10^{-3}$ and $6.97 \times 10^{-4} \mathrm{~kg}$ PM2.5 eq , which account for about $99.8 \%$ of the total respiratory effects caused by inorganics. Both the CFs of human toxicity and respiratory effects could be normalized to the disability-adjusted life years through suitable normalization factors to make them comparable [20].

Terrestrial ecotoxicity effects were determined by dividing the damage $\mathrm{CF}$ of the reference substance triethylene glycol into soil (kg TEG soil) in the IMPACT 2002+ model [19]. It has been estimated that the substances have ecotoxic effects only by exposure through the aqueous phase in soil. The terrestrial ecotoxicity damage from SUB2 was about $0.431 \mathrm{~kg}$ TEG soil or $46.2 \mathrm{PDF} \mathrm{m}^{2}$ per year after normalization.

Global warming effects were expressed in kilogram equivalents of carbon dioxide into the air $\left(\mathrm{kg} \mathrm{CO}_{2-\mathrm{eq}}\right.$ into 
Table 3 Midpoint impacts of SCWD for $1 \mathrm{~kg}$ PFOS waste treatment in a lab-scale batch operation

\begin{tabular}{|c|c|c|c|c|c|c|c|c|c|}
\hline Impact category & Unit & $\begin{array}{l}\text { SUB1-Ar and iron } \\
\text { powder preparation }\end{array}$ & $\%$ & $\begin{array}{l}\text { SUB2-heat } \\
\text { supply }\end{array}$ & $\%$ & $\begin{array}{l}\text { SUB3-CHF } \\
\text { emission }\end{array}$ & $\%$ & $\begin{array}{l}\text { SUB4-F } \\
\text { wastewater } \\
\text { treatment }\end{array}$ & $\%$ \\
\hline Carcinogens & DALY & $4.58 \mathrm{E}-11$ & 0.13 & $3.39 \mathrm{E}-08$ & 99.72 & - & - & $5.07 \mathrm{E}-11$ & 0.15 \\
\hline Non-carcinogens & DALY & $6.67 \mathrm{E}-11$ & 0.11 & $5.78 \mathrm{E}-08$ & 99.07 & - & - & $4.76 \mathrm{E}-10$ & 0.82 \\
\hline $\begin{array}{l}\text { Respiratory } \\
\text { inorganics }\end{array}$ & DALY & $2.17 \mathrm{E}-09$ & 0.06 & $3.83 \mathrm{E}-06$ & 99.92 & - & - & $8.05 \mathrm{E}-10$ & 0.02 \\
\hline Ionizing radiation & DALY & $4.94 \mathrm{E}-11$ & 2.36 & $2.04 \mathrm{E}-09$ & 97.39 & - & - & $5.16 \mathrm{E}-12$ & 0.25 \\
\hline $\begin{array}{c}\text { Ozone layer } \\
\text { depletion }\end{array}$ & DALY & $2.63 \mathrm{E}-13$ & 0.04 & $6.10 \mathrm{E}-10$ & 99.93 & - & - & $1.54 \mathrm{E}-13$ & 0.03 \\
\hline $\begin{array}{c}\text { Respiratory } \\
\text { organics }\end{array}$ & DALY & $1.61 \mathrm{E}-12$ & 0.07 & $2.38 \mathrm{E}-09$ & 99.88 & - & - & $1.26 \mathrm{E}-12$ & 0.05 \\
\hline $\begin{array}{l}\text { Aquatic } \\
\text { ecotoxicity }\end{array}$ & $\mathrm{PDF} \mathrm{m}^{2}$ year & $9.31 \mathrm{E}-06$ & 0.09 & $9.78 \mathrm{E}-03$ & 98.49 & - & - & $1.41 \mathrm{E}-04$ & 1.42 \\
\hline $\begin{array}{l}\text { Terrestrial } \\
\text { ecotoxicity }\end{array}$ & PDF $\mathrm{m}^{2}$ year & $3.30 \mathrm{E}-04$ & 0.08 & $4.31 \mathrm{E}-01$ & 98.64 & - & - & $5.61 \mathrm{E}-03$ & 1.28 \\
\hline $\begin{array}{c}\text { Terrestrial acid/ } \\
\text { neutralization }\end{array}$ & PDF $\mathrm{m}^{2}$ year & $6.18 \mathrm{E}-05$ & 0.05 & $1.25 \mathrm{E}-01$ & 99.92 & - & - & $3.46 \mathrm{E}-05$ & 0.03 \\
\hline Land occupation & PDF $\mathrm{m}^{2}$ year & $1.54 \mathrm{E}-05$ & 0.28 & $5.37 \mathrm{E}-03$ & 99.23 & - & - & $2.63 \mathrm{E}-05$ & 0.49 \\
\hline Global warming & $\mathrm{kg} \mathrm{CO} 2$-eq & $3.74 \mathrm{E}-03$ & 0.08 & $4.81 \mathrm{E}+00$ & 99.60 & $1.47 \mathrm{E}-02$ & 0.30 & $6.93 \mathrm{E}-04$ & 0.01 \\
\hline $\begin{array}{l}\text { Non-renewable } \\
\text { energy }\end{array}$ & MJ primary & $7.40 \mathrm{E}-02$ & 0.11 & $6.87 \mathrm{E}+01$ & 99.87 & - & - & $1.56 \mathrm{E}-02$ & 0.02 \\
\hline Mineral extraction & MJ primary & $1.90 \mathrm{E}-05$ & 0.41 & $4.53 \mathrm{E}-03$ & 98.46 & - & - & $5.18 \mathrm{E}-05$ & 1.13 \\
\hline
\end{tabular}

"DALY" ("Disability-Adjusted Life Years") characterizes the disease severity, accounting for both mortality (years of life lost due to premature death) and morbidity (the time of life with lower quality due to an illness, e.g., at hospital); "PDF $\mathrm{m}^{2}$ year" ("Potentially Disappeared Fraction of species over a certain amount of $\mathrm{m}^{2}$ during a certain amount of the year") is the unit to measure" the impacts on ecosystems; MJ ("Mega Joules") measures the amount of energy extracted or needed to extract the resource

air) and taken from the IPCC list (IPCC 2001, and IPCC 2007 for $\mathrm{CH}_{4}, \mathrm{~N}_{2} \mathrm{O}$ and $\mathrm{CO}$ ). The Global Warming Potentials (GWPs) for a 100-year time horizon are used [21]. The major global warming substances considered in SUB2 are carbon dioxide, dinitrogen monoxide, methane, carbon monoxide, sulfur hexafluoride, methane, and ethane . The carbon dioxide emission from the oil burning for electric generation was about $4.81 \mathrm{~kg}$ for the treatment of $1 \mathrm{~kg}$ of PFOS waste, which takes more than $99 \%$ of the whole contributions in SUB2, as shown in Table 6. And the contribution of $\mathrm{CHF}_{3}$ emission in SUB3 is only $0.0147 \mathrm{~kg}$ $\mathrm{CO}_{2 \text {-eq. }}$

Non-renewable energy consumption effects, in terms of the total primary energy extracted, were expressed in mega Joules of total primary non-renewable energy/unit extracted and taken from the Ecoinvent system. The nonrenewable energy consumption in SUB2 was mainly from the crude oil and natural gas usage, which accounts to 63.7 and $2.98 \mathrm{MJ}$ for the treatment of $1 \mathrm{~kg}$ of PFOS waste, respectively.

Mineral extraction effects were expressed in mega Joules of surplus energy per kilogram extracted and taken directly from Eco-indicator 99 [16]. Surplus energy expresses the expected increase of extraction energy needed to extract 5 times the cumulative extracted amount since the beginning of extraction until 1990. The total mineral extraction damage in SUB2 is about $0.0046 \mathrm{MJ}$ surplus.

The normalization was performed in Impact $2002+$ by dividing the impact (at damage categories) by the respective normalization factors. The unit of "point (pt)" is equal to "person year" in the SimaPro, which was used to express the respective share of each impact normalized to the overall damage of the considered category. The normalization factors for the four damage categories are 0.0071 DALY/point for human health, 13,800 PDF $\mathrm{m}^{2}$ year/point for ecosystem quality, $11,600 \mathrm{~kg} \mathrm{CO}$ into air/point for climate change and 152,000 MJ/point for resources. A "pt" represents the average impact in a specific category caused by a person during one year in Europe. For human health, it also represents the average impact on a person during one year. It is calculated as the total yearly damage score due to emissions and extractions in Europe divided by the total European population.

More details could be found from the normalized midpoint scores of subsystems in Fig. 3. It could be found that most of the negative impacts are related to global warming, non-renewable energy, respiratory inorganics, and 
Table 4 The carcinogenic substance emissions from $1 \mathrm{~kg}$ PFOS waste treatment by SCWD in a lab-scale batch operation $\left(\mathrm{Unit}_{\mathrm{kg}} \mathrm{C}_{2} \mathrm{H}_{3} \mathrm{Cl}_{\text {-eq }}\right)$

\begin{tabular}{|c|c|c|c|c|c|c|c|}
\hline$\overline{\mathrm{No}}$ & Substances & Compartment & Total & $\begin{array}{l}\text { SUB1-Ar \& iron } \\
\text { powder preparation }\end{array}$ & $\begin{array}{l}\text { SUB2-heat } \\
\text { supply }\end{array}$ & $\begin{array}{l}\text { SUB3-CHF }_{3} \\
\text { emission }\end{array}$ & $\begin{array}{l}\text { SUB4-F } \\
\text { wastewater } \\
\text { treatment }\end{array}$ \\
\hline 1 & Hydrocarbons, aromatic & Air & $4.44 \mathrm{E}-03$ & $1.02 \mathrm{E}-05$ & $4.42 \mathrm{E}-03$ & $\times$ & $8.84 \mathrm{E}-06$ \\
\hline 2 & $\begin{array}{l}\text { Dioxin, } 2,3,7,8 \\
\text { Tetrachlorodibenzo-p- }\end{array}$ & Air & $1.18 \mathrm{E}-03$ & $6.74 \mathrm{E}-07$ & $1.17 \mathrm{E}-03$ & $\times$ & $2.91 \mathrm{E}-06$ \\
\hline 3 & Arsenic & Air & $5.71 \mathrm{E}-04$ & $6.06 \mathrm{E}-07$ & $5.70 \mathrm{E}-04$ & $x$ & $1.78 \mathrm{E}-07$ \\
\hline 4 & $\begin{array}{l}\text { PAH, polycyclic aromatic } \\
\text { hydrocarbons }\end{array}$ & Air & $5.38 \mathrm{E}-04$ & $7.52 \mathrm{E}-07$ & $5.36 \mathrm{E}-04$ & $x$ & $7.08 \mathrm{E}-07$ \\
\hline 5 & Benzo(a)pyrene & Air & $1.17 \mathrm{E}-04$ & $1.69 \mathrm{E}-06$ & $1.15 \mathrm{E}-04$ & $x$ & $1.44 \mathrm{E}-07$ \\
\hline 6 & Chromium & Air & $1.10 \mathrm{E}-04$ & $1.31 \mathrm{E}-07$ & $1.09 \mathrm{E}-04$ & $x$ & $4.83 \mathrm{E}-07$ \\
\hline 7 & Formaldehyde & Air & $3.72 \mathrm{E}-05$ & $3.30 \mathrm{E}-08$ & $3.72 \mathrm{E}-05$ & $x$ & $5.13 \mathrm{E}-09$ \\
\hline 8 & Cadmium & Air & $8.72 \mathrm{E}-06$ & $3.90 \mathrm{E}-09$ & $8.71 \mathrm{E}-06$ & $x$ & $1.44 \mathrm{E}-09$ \\
\hline 9 & Chromium VI & Air & $2.66 \mathrm{E}-06$ & $6.32 \mathrm{E}-09$ & $2.64 \mathrm{E}-06$ & $x$ & $1.23 \mathrm{E}-08$ \\
\hline 10 & Acetaldehyde & Air & $9.10 \mathrm{E}-07$ & $1.18 \mathrm{E}-09$ & $9.08 \mathrm{E}-07$ & $x$ & $2.01 \mathrm{E}-10$ \\
\hline 11 & Phenol, pentachloro- & Air & $1.11 \mathrm{E}-07$ & $2.63 \mathrm{E}-09$ & $1.09 \mathrm{E}-07$ & $x$ & $8.12 \mathrm{E}-11$ \\
\hline 12 & Beryllium & Air & $7.78 \mathrm{E}-08$ & $1.21 \mathrm{E}-10$ & $7.77 \mathrm{E}-08$ & $x$ & $2.47 \mathrm{E}-11$ \\
\hline 13 & Arsenic & Soil & $1.65 \mathrm{E}-04$ & $6.31 \mathrm{E}-08$ & $1.61 \mathrm{E}-04$ & $\times$ & $3.11 \mathrm{E}-06$ \\
\hline 14 & Hydrocarbons, aromatic & Water & $4.80 \mathrm{E}-03$ & $1.12 \mathrm{E}-06$ & $4.80 \mathrm{E}-03$ & $x$ & $7.28 \mathrm{E}-07$ \\
\hline 15 & Arsenic, ion & Water & $1.29 \mathrm{E}-04$ & $1.02 \mathrm{E}-06$ & $1.27 \mathrm{E}-04$ & $\times$ & $9.83 \mathrm{E}-07$ \\
\hline 16 & $\begin{array}{l}\text { PAH, polycyclic aromatic } \\
\text { hydrocarbons }\end{array}$ & Water & $4.37 \mathrm{E}-05$ & $1.08 \mathrm{E}-08$ & $4.37 \mathrm{E}-05$ & $x$ & $6.94 \mathrm{E}-09$ \\
\hline 17 & Toluene & Water & $4.60 \mathrm{E}-07$ & $1.08 \mathrm{E}-10$ & $4.60 \mathrm{E}-07$ & $x$ & $7.19 \mathrm{E}-11$ \\
\hline 18 & Benzene & Water & $4.44 \mathrm{E}-07$ & $1.12 \mathrm{E}-10$ & $4.44 \mathrm{E}-07$ & $x$ & $1.17 \mathrm{E}-10$ \\
\hline 19 & Methane, dichloro-, HCC-30 & Water & $2.09 \mathrm{E}-07$ & $5.14 \mathrm{E}-11$ & $2.09 \mathrm{E}-07$ & $x$ & $1.92 \mathrm{E}-11$ \\
\hline 20 & Benzene, ethyl- & Water & $5.96 \mathrm{E}-08$ & $1.39 \mathrm{E}-11$ & $5.96 \mathrm{E}-08$ & $\times$ & $8.99 \mathrm{E}-12$ \\
\hline
\end{tabular}

terrestrial ecotoxicity. The human health impact from carcinogens and non-carcinogens pollutant emission and terrestrial acid and neutralization should also be considered. Nearly all of those environmental impacts arose from the fuel burning to supply enough heat in SUB2 to sustain a subcritical condition at high temperature and high pressure. Other impacts such as ionizing radiation, ozone layer depletion, respiratory organics, aquatic ecotoxicity, land occupation and mineral extraction are three orders of magnitude lower than the global warming effect.

The midpoint impact categories can also be grouped into four end-point categories. Those four end-point categories were also calculated by respective damage factors and shown in Fig. 4. We can also compare the different endpoint environmental damages between different subsystems. These results were normalized by comparing them against the environmental profile of an "average European", which is embedded in the IMPACT 2002+ LCIA methodology in SimaPro 7.3. The results of end-point damage were also shown in the dimensionless unit "pt", together with a summed total score. It could be found that the impacts to human health, climate change and resources are the major environmental impacts from the SCWD of
PFOS waste. The ecological quality impact is one order of magnitude lower than the climate change impact.

Life cycle impact assessment interpretation

To interpret the environmental impacts precisely, we calculated the endpoint impacts of the four subsystems of SCWD technology. The total negative impact to the human health, ecological quality, climate change and resources accounts to $1.11 \times 10^{-3}, 8.43 \times 10^{-5}, 9.76 \times 10^{-4}$, $9.05 \times 10^{-4} \mathrm{Pt}$ for a $\mathrm{kg}$ of PFOS waste, respectively. It could be found that most of the endpoint impacts were also generated by SUB2. That just shows the characteristics of a large amount fuel consumption in a subcritical water treatment process.

Figure 5 shows the process specific environmental impact of the SCWD system for PFOS waste treatment. It could be found that the SUB2-heat supply for the SCWD system contributes nearly all of the environmental impacts (99.8\%). Also, the argon consumption was also the major source of the environmental impact in a lab-scale batch subcritical water decomposition operation for the treatment of PFOS waste. When compared with other industrialized 
Table 5 The non-carcinogenic substance emissions from $1 \mathrm{~kg}$ PFOS waste treatment by SCWD in a lab-scale batch operation (Unit: kg $\mathrm{C}_{2} \mathrm{H}_{3} \mathrm{Cl}_{\text {-eq }}$ )

\begin{tabular}{|c|c|c|c|c|c|c|c|}
\hline No & Substances & Compartment & Total & $\begin{array}{l}\text { SUB1-Ar and iron } \\
\text { powder preparation }\end{array}$ & $\begin{array}{l}\text { SUB2-Heat } \\
\text { supply }\end{array}$ & $\begin{array}{l}\mathrm{SUB}-\mathrm{CHF}_{3} \\
\text { emission }\end{array}$ & $\begin{array}{l}\text { SUB4-F- } \\
\text { wastewater } \\
\text { treatment }\end{array}$ \\
\hline 1 & Dioxin, 2,3,7,8 Tetrachlorodibenzo-p- & Air & $5.94 \mathrm{E}-03$ & $3.40 \mathrm{E}-06$ & $5.92 \mathrm{E}-03$ & $\times$ & $1.47 \mathrm{E}-05$ \\
\hline 2 & Arsenic & Air & $5.73 \mathrm{E}-03$ & $6.08 \mathrm{E}-06$ & $5.72 \mathrm{E}-03$ & $\times$ & $1.78 \mathrm{E}-06$ \\
\hline 3 & Molybdenum & Air & $8.16 \mathrm{E}-04$ & $2.75 \mathrm{E}-07$ & $8.16 \mathrm{E}-04$ & $\times$ & $1.86 \mathrm{E}-08$ \\
\hline 4 & Zinc & Air & $1.71 \mathrm{E}-04$ & $1.84 \mathrm{E}-07$ & $1.70 \mathrm{E}-04$ & $x$ & $1.18 \mathrm{E}-07$ \\
\hline 5 & Nickel & Air & $1.58 \mathrm{E}-04$ & $3.29 \mathrm{E}-08$ & $1.58 \mathrm{E}-04$ & $\times$ & $3.55 \mathrm{E}-09$ \\
\hline 6 & Formaldehyde & Air & $1.34 \mathrm{E}-04$ & $1.19 \mathrm{E}-07$ & $1.34 \mathrm{E}-04$ & $x$ & $1.85 \mathrm{E}-08$ \\
\hline 7 & Cadmium & Air & $1.34 \mathrm{E}-04$ & $6.00 \mathrm{E}-08$ & $1.34 \mathrm{E}-04$ & $\times$ & $2.21 \mathrm{E}-08$ \\
\hline 8 & Benzene & Air & $8.69 \mathrm{E}-05$ & $1.13 \mathrm{E}-07$ & $8.68 \mathrm{E}-05$ & $x$ & $1.85 \mathrm{E}-08$ \\
\hline 9 & Selenium & Air & $6.13 \mathrm{E}-05$ & $5.26 \mathrm{E}-08$ & $6.12 \mathrm{E}-05$ & $x$ & $3.91 \mathrm{E}-09$ \\
\hline 10 & Chromium & Air & $1.62 \mathrm{E}-05$ & $1.93 \mathrm{E}-08$ & $1.61 \mathrm{E}-05$ & $x$ & $7.13 \mathrm{E}-08$ \\
\hline 11 & Mercury & Air & $1.44 \mathrm{E}-05$ & $4.68 \mathrm{E}-08$ & $1.44 \mathrm{E}-05$ & $x$ & $2.21 \mathrm{E}-08$ \\
\hline 12 & Arsenic & Soil & $1.71 \mathrm{E}-03$ & $6.56 \mathrm{E}-07$ & $1.68 \mathrm{E}-03$ & $x$ & $3.23 \mathrm{E}-05$ \\
\hline 13 & Barium & Soil & $1.05 \mathrm{E}-03$ & $2.58 \mathrm{E}-07$ & $1.05 \mathrm{E}-03$ & $\times$ & $9.65 \mathrm{E}-08$ \\
\hline 14 & Zinc & Soil & $6.13 \mathrm{E}-04$ & $7.40 \mathrm{E}-07$ & $5.22 \mathrm{E}-04$ & $x$ & $9.03 \mathrm{E}-05$ \\
\hline 15 & Aluminium & Soil & $1.57 \mathrm{E}-04$ & $5.42 \mathrm{E}-08$ & $1.52 \mathrm{E}-04$ & $x$ & $5.53 \mathrm{E}-06$ \\
\hline 16 & Molybdenum & Soil & $1.23 \mathrm{E}-05$ & $1.98 \mathrm{E}-08$ & $8.95 \mathrm{E}-07$ & $\times$ & $1.14 \mathrm{E}-05$ \\
\hline 17 & Barium & Water & $2.56 \mathrm{E}-03$ & $6.01 \mathrm{E}-07$ & $2.56 \mathrm{E}-03$ & $\times$ & $3.87 \mathrm{E}-07$ \\
\hline 18 & Arsenic, ion & Water & $1.34 \mathrm{E}-03$ & $1.07 \mathrm{E}-05$ & $1.32 \mathrm{E}-03$ & $\times$ & $1.02 \mathrm{E}-05$ \\
\hline 19 & Cadmium, ion & Water & $1.86 \mathrm{E}-05$ & $1.38 \mathrm{E}-08$ & $1.85 \mathrm{E}-05$ & $\times$ & $1.13 \mathrm{E}-07$ \\
\hline 20 & Nickel, ion & Water & $1.07 \mathrm{E}-05$ & $4.08 \mathrm{E}-09$ & $1.07 \mathrm{E}-05$ & $x$ & $4.50 \mathrm{E}-08$ \\
\hline
\end{tabular}

waste treatment facilities, such as the high temperature incineration and base catalyzed decomposition for POPs waste disposal with environmental impacts of 0.279 and $0.147 \mathrm{Pt} /$ ton PCBs waste, this lab scale SCWD reactor consumed too much energy to sustain the subcritical condition $\left(350{ }^{\circ} \mathrm{C}\right.$ and $\left.23 \mathrm{MPa}\right)$ for the PFOS waste disposal, which contributes the major negative environmental impacts in the operation [22, 23].

To improve the energy efficiency is one of the most important factors to reduce the environmental impact when sustaining the high temperature and high pressure from the SCWD process, such as heat recovery or using clean energy sources. When compared with the batch operation, the continuous operation could also improve the energy efficiency and reduce the heat loss in the SCWD operation. The improvement of catalyst is also necessary to shorten the reaction time and increase the system effectiveness for the PFOS waste treatment through the SCWD technology.

\section{Uncertainty analysis}

Uncertainties can occur in the inventory and impact assessment calculation in this study. For instance, the inventory for both SCWD and the electric iron powder process was mainly from the published papers, unpublished data might have some impacts on the LCA of the whole process. Also, the inventories for some basic processes supplied by the Ecoinvent Database and adopted by this study have some uncertainties. There are also some uncertainties in the impact analysis approached in the IMPACT2002+ [16]. Generally speaking, uncertainties in inventory are high compared to uncertainties in impact analysis, when considering that most of the technological parameters were taken from a lab-scale batch SCWD operation. A detailed quantitative uncertainty analysis was not covered in this preliminary LCA study, which was supposed to be more meaningful for the improved and commercialized SCWD operation.

\section{Conclusions}

The IMPACT 2002+ environmental model was used to evaluate the environmental impact of a lab-scale batch subcritical water decomposition operation for a $\mathrm{kg}$ of PFOS waste treatment in this study. The total negative impacts of the SCWD process to the human health, ecological quality, 
Table 6 The global warming substance emissions from $1 \mathrm{~kg}$ PFOS waste treatment by SCWD in a lab-scale batch operation $\left(\mathrm{Unit}_{\mathrm{kg}} \mathrm{CO}_{2}\right.$-eq $)$

\begin{tabular}{|c|c|c|c|c|c|c|c|}
\hline No & Substances & Compartment & Total & $\begin{array}{l}\text { SUB1-Ar and iron } \\
\text { powder preparation }\end{array}$ & $\begin{array}{l}\text { SUB2-Heat } \\
\text { supply }\end{array}$ & $\begin{array}{l}\text { SUB3-CHF } 3 \\
\text { emission }\end{array}$ & $\begin{array}{l}\text { SUB4-F } \\
\text { wastewater } \\
\text { treatment }\end{array}$ \\
\hline 1 & Carbon dioxide, fossil & Air & $4.75 \mathrm{E}+00$ & $3.51 \mathrm{E}-03$ & $4.75 \mathrm{E}+00$ & $\times$ & $6.51 \mathrm{E}-04$ \\
\hline 2 & Dinitrogen monoxide & Air & $3.71 \mathrm{E}-02$ & $1.88 \mathrm{E}-05$ & $3.71 \mathrm{E}-02$ & $x$ & $1.82 \mathrm{E}-05$ \\
\hline 3 & Methane, fossil & Air & $2.75 \mathrm{E}-02$ & $6.03 \mathrm{E}-05$ & $2.74 \mathrm{E}-02$ & $\times$ & $1.53 \mathrm{E}-05$ \\
\hline 4 & Methane, trifluoro-, HFC-23 & Air & $1.47 \mathrm{E}-02$ & $1.65 \mathrm{E}-10$ & $1.17 \mathrm{E}-08$ & $1.47 \mathrm{E}-02$ & $8.92 \mathrm{E}-11$ \\
\hline 5 & Carbon monoxide, fossil & Air & $2.66 \mathrm{E}-03$ & $5.53 \mathrm{E}-06$ & $2.65 \mathrm{E}-03$ & $\times$ & $3.19 \mathrm{E}-06$ \\
\hline 6 & Sulfur hexafluoride & Air & $5.19 \mathrm{E}-04$ & $1.23 \mathrm{E}-05$ & $5.07 \mathrm{E}-04$ & $x$ & $5.92 \mathrm{E}-07$ \\
\hline 7 & Methane, bromotrifluoro-, Halon 1301 & Air & $1.29 \mathrm{E}-04$ & $3.21 \mathrm{E}-08$ & $1.29 \mathrm{E}-04$ & $x$ & $2.73 \mathrm{E}-08$ \\
\hline 8 & Carbon dioxide & Air & $1.25 \mathrm{E}-04$ & $1.25 \mathrm{E}-04$ & $x$ & $\times$ & $\times$ \\
\hline 9 & Methane, tetrafluoro-, CFC-14 & Air & $1.03 \mathrm{E}-04$ & $1.30 \mathrm{E}-06$ & $1.01 \mathrm{E}-04$ & $x$ & $4.41 \mathrm{E}-07$ \\
\hline 10 & Methane, biogenic & Air & $4.95 \mathrm{E}-05$ & $9.88 \mathrm{E}-07$ & 4.47E-05 & $\times$ & $3.89 \mathrm{E}-06$ \\
\hline 11 & Ethane, hexafluoro-, HFC-116 & Air & $2.36 \mathrm{E}-05$ & $2.96 \mathrm{E}-07$ & $2.32 \mathrm{E}-05$ & $x$ & $1.04 \mathrm{E}-07$ \\
\hline 12 & Carbon dioxide, land transformation & Air & $2.11 \mathrm{E}-05$ & $2.04 \mathrm{E}-07$ & $2.09 \mathrm{E}-05$ & $x$ & $8.61 \mathrm{E}-09$ \\
\hline 13 & $\begin{array}{l}\text { Ethane, 1,2-dichloro-1,1,2,2-tetrafluoro-, } \\
\text { CFC-114 }\end{array}$ & Air & $1.09 \mathrm{E}-05$ & $2.56 \mathrm{E}-07$ & $1.06 \mathrm{E}-05$ & $x$ & $3.36 \mathrm{E}-08$ \\
\hline 14 & Methane & Air & $9.92 \mathrm{E}-06$ & $9.92 \mathrm{E}-06$ & $x$ & $x$ & $\times$ \\
\hline 15 & Ethane, 1,1,1,2-tetrafluoro-, HFC-134a & Air & $6.08 \mathrm{E}-06$ & $7.03 \mathrm{E}-09$ & $5.94 \mathrm{E}-06$ & $\times$ & $1.30 \mathrm{E}-07$ \\
\hline 16 & Methane, chlorodifluoro-, HCFC-22 & Air & $2.04 \mathrm{E}-06$ & $3.38 \mathrm{E}-08$ & $2.00 \mathrm{E}-06$ & $\times$ & $1.84 \mathrm{E}-09$ \\
\hline 17 & Methane, tetrachloro, CFC-10 & Air & $4.12 \mathrm{E}-07$ & $4.95 \mathrm{E}-10$ & $3.99 \mathrm{E}-07$ & $x$ & $1.30 \mathrm{E}-08$ \\
\hline 18 & Methane, bromochlorodifluoro, Halon 1211 & Air & $3.72 \mathrm{E}-07$ & 4.95E-09 & $3.67 \mathrm{E}-07$ & $x$ & $3.09 \mathrm{E}-10$ \\
\hline 19 & Carbon monoxide & Air & $1.59 \mathrm{E}-07$ & $1.59 \mathrm{E}-07$ & $x$ & $\times$ & $\times$ \\
\hline 20 & Methane, dichlorodifluoro, CFC-12 & Air & $5.51 \mathrm{E}-08$ & $4.18 \mathrm{E}-10$ & $5.46 \mathrm{E}-08$ & $x$ & $7.76 \mathrm{E}-11$ \\
\hline
\end{tabular}

Fig. 3 Normalized midpoint impacts of SCWD for $1 \mathrm{~kg}$ of PFOS waste treatment in a labscale batch operation

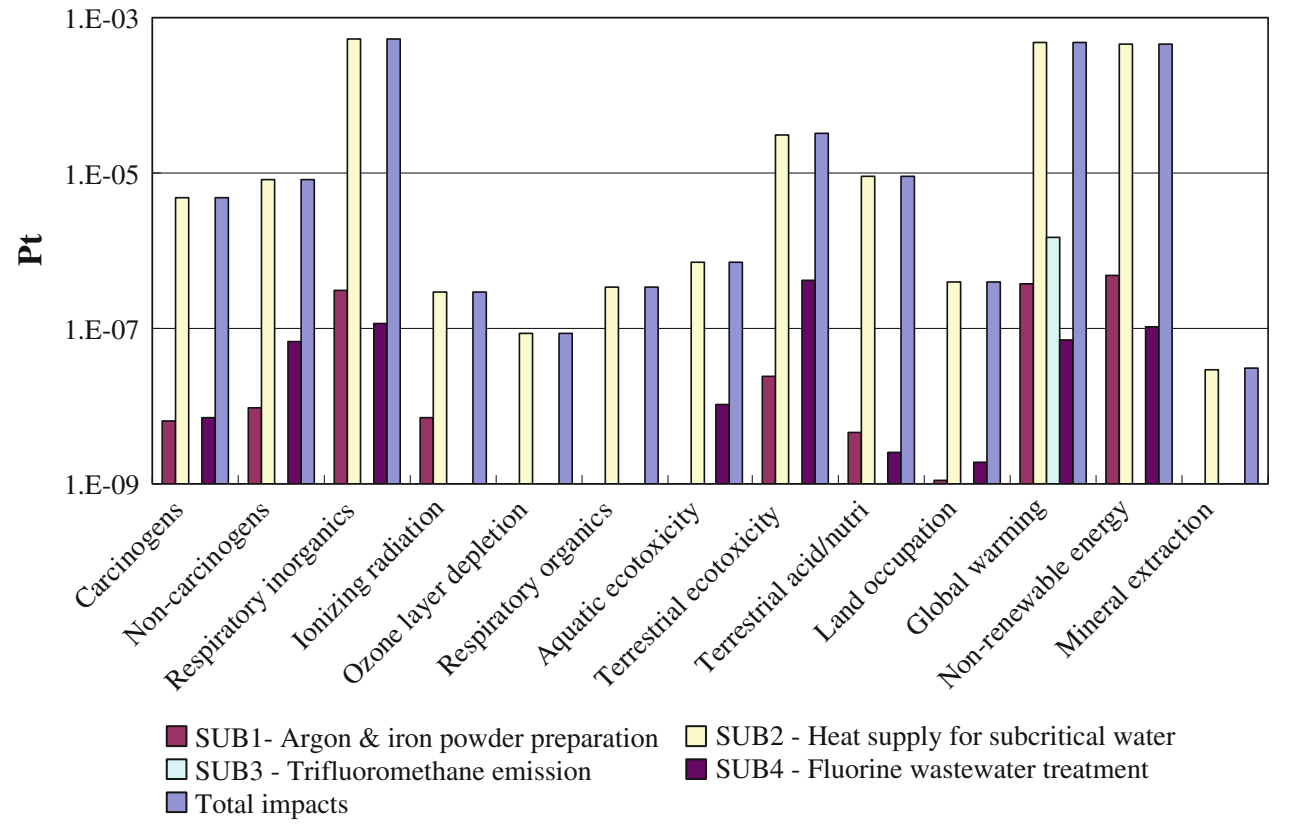

climate change and resources are about $1.11 \times 10^{-3}$, $8.43 \times 10^{-5}, 9.76 \times 10^{-4}, 9.05 \times 10^{-4}$ Pt per kg of PFOS waste, respectively. It was found that the energy consumption to sustain the high temperature and high pressure in the subcritical water process contributes the most part of the environmental impact. And the improvement of energy efficiency and catalytic effectiveness is a potential way to reduce the environmental impacts from the SCWD process. 
Fig. 4 Comparison of normalized endpoint scores of subsystems of the SCWD for $1 \mathrm{~kg}$ of PFOS waste treatment in a lab-scale batch operation

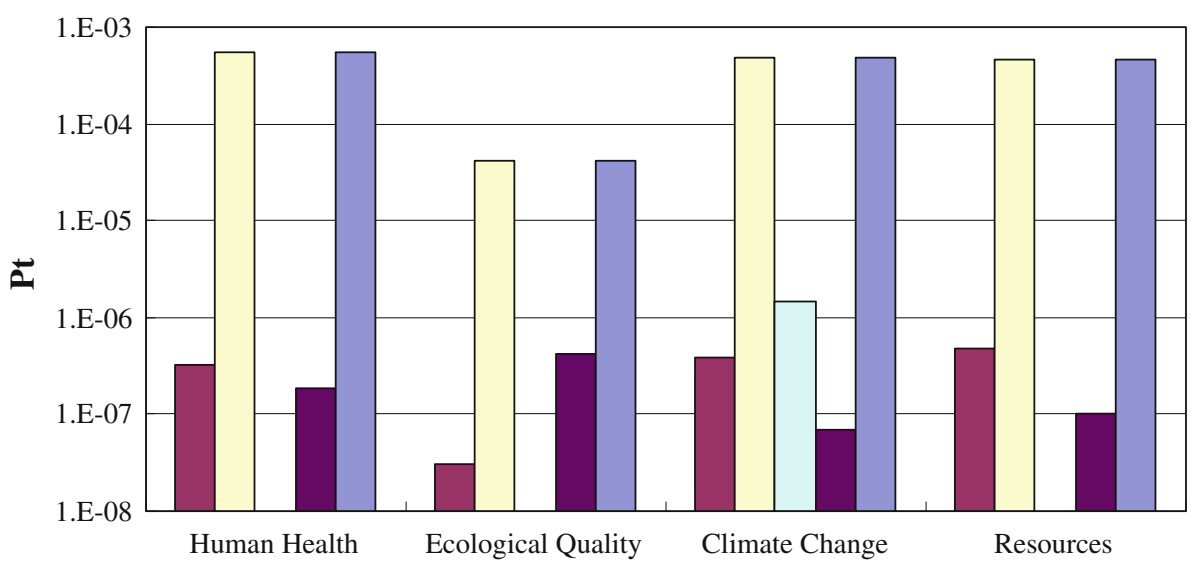

$\square$ SUB1- Argon \& iron powder preparation $\square$ SUB3 - Trifluoromethane emission $\square$ Total impacts

$\square$ SUB2 - Heat supply for subcritical water SUB4 - Fluorine wastewater treatment

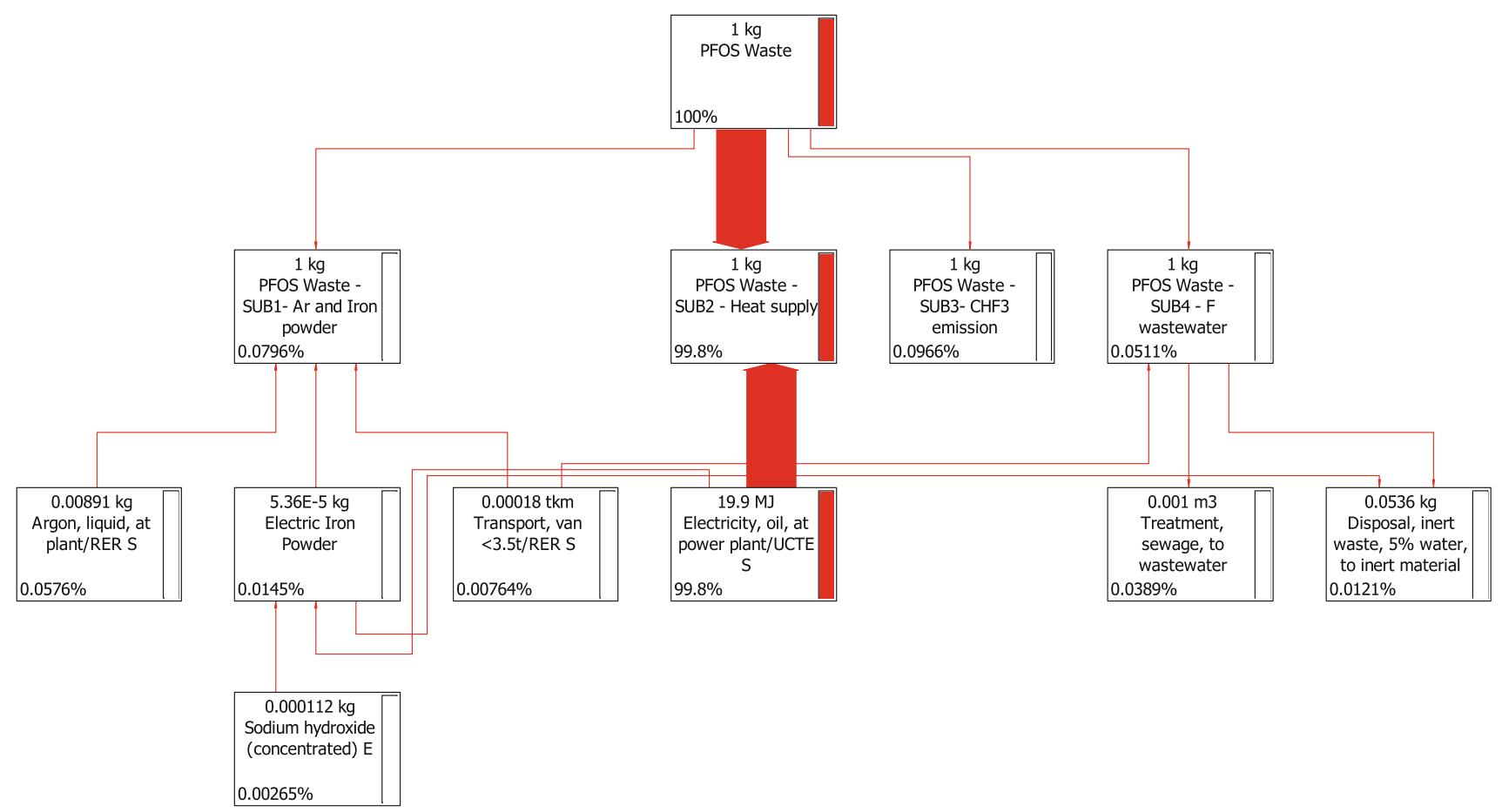

Fig. 5 Process environmental impact of the SCWD for $1 \mathrm{~kg}$ of PFOS waste treatment in a lab-scale batch operation

The LCA analysis was entirely based on European conditions and adopted without change for this study, so the conclusions are also strictly limited to this context, which may result in uncertainties because of data availability, system boundaries and the choice of impact analysis method.

Acknowledgments Support for this research was provided by the National Science Foundation of China (20977105 and 50708110) and the Environmental Public Welfare Project (201009026) and is gratefully acknowledged. We also want to thank PRé Consultants for providing the LCA software SIMARPO for this study.

\section{References}

1. Renner R (2008) PFOS phaseout pays off. Environ Sci Technol 42:4618-4618. doi:10.1021/es0871614

2. Liu C, Hu J, Liu J (2008) Release of perfluorooctane sulfonate (PFOS) from semiconductor manufacturing site and environmental concentration estimation and environmental risk assessment of PFOS near semiconductor manufacturing site. Sci Tech Engng 8:2898-2902. Available at http://en.cnki.com.cn/Article_ en/CJFDTOTAL-KXJS200811032.htm

3. Paul AG, Jones KC, Sweetman AJ (2008) A first global production, emission, and environmental inventory for perfluorooctane sulfonate. Environ Sci Technol 43:386-392. doi:10.1021/es802216n 
4. Pan G, Jia CX, Zhao DY, You C, Chen H, Jiang GB (2009) Effect of cationic and anionic surfactants on the sorption and desorption of perfluorooctane sulfonate (PFOS) on natural sediments. Environ Pollut 15:325-330. doi:10.1016/j.envpol.2008.06.035

5. Zhao YG, Wong CKC, Wong MH (2012) Environmental contamination, human exposure and body loadings of perfluorooctane sulfonate (PFOS), focusing on Asian countries. Chemosphere 89:355-368. doi:10.1016/j.chemosphere.2012.05.043

6. Wang T, Khim JS, Chen C (2011) Perfluorinated compounds in surface waters from Northern China: Comparison to level of industrialization. Environ Int 42(S):37-46. doi:10.1016/j.envint. 2011.03.023

7. Suominen K, Hallikainen A, Ruokojarvi P (2011) Occurrence of PCDD/F, PCB, PBDE, PFAS, and organotin compounds in fish meal, fish oil and fish feed. Chemosphere 85:300-306. doi: 10.1016/j.chemosphere.2011.06.010

8. Lindstrom AB, Strynar MJ, Libelo EL (2011) Polyfluorinated compounds: past, present, and future. Environ Sci Technol 45:7954-7961. doi:10.1021/es2011622

9. Vecitis CD, Park H, Cheng J, Mader BT, Hoffmann MR (2009) Treatment technologies for aqueous perfluorooctanesulfonate (PFOS) and perfluorooctanoate (PFOA). Front Environ Sci Eng 3:129-151. doi:10.1007/s11783-009-0022-7

10. Tang CYY, Fu QS, Robertson AP, Criddle CS, Leckie JO (2006) Use of reverse osmosis membranes to remove perfluorooctane sulfonate (PFOS) from semiconductor wastewater. Environ Sci Technol 23:7343-7349. doi:10.1021/Es060831q

11. Hori H, Nagaoka Y, Yamamoto A (2006) Efficient decomposition of environmentally persistent perfluorooctanesulfonate and related fluorochemicals using zerovalent iron in subcritical water. Environ Sci Technol 40:1049-1054. doi:101021/Es0517419

12. Rebitzer G, Ekvall T, Frischknecht R (2004) Life cycle assessment Part 1: framework, goal and scope definition, inventory analysis, and applications. Environ Int 30:701-720. doi:10. 1016/j.envint.2003.11.005

13. Ekvall T, Weidema BP (2004) System boundaries and input data in consequential life cycle inventory analysis. Int J Life Cycle Ass 9:161-171. doi:10.1065/lca2004.03.148
14. Suer P, Nilsson-Paledal S, Norrman J (2004) LCA for site remediation: a literature review. Soil Sediment Contam 13:415-425. doi:10.1080/10588330490471304

15. Diamond ML, Page CA, Campbell M (1999) Life-cycle framework for assessment of site remediation options: method and generic survey. Environ Toxicol Chem 18:788-800. doi:10.1897/ 1551-5028(1999)018<0788:LCFFAO $>2.3$. CO;2

16. Jolliet O, Margni M, Charles R (2003) IMPACT 2002+: a new life cycle impact assessment methodology. Int J Life Cycle Ass 8:324-330. doi:10.1007/BF02978505

17. Cinici H, Karacif K, Kafkas F (2011) Effect of electrolytic nickel coating on fatigue life of iron based powder metal parts. Kovove Mater 49:355-359. doi:10.4149/km 2011 5 355

18. Pennington DW, Margni M, Amman C, Jolliet O (2005) Spatial versus non-spatial multimedia fate and exposure modeling: insights for Western Europe. Environ Sci Technol 39:1119-1128. doi:10.1021/es034598x

19. Dockery DW, Pope CA (1994) Acute respiratory effects of particulate pollution. Annu Rev Publ Health 15:107-132. doi: 10.1146/annurev.publhealth.15.1.107

20. Crettaz P, Pennington D, Rhomberg L, Brand B, Jolliet O (2002) Assessing human health response in life cycle assessment using ED10s and DALYs: part 1-cancer effects. Risk Anal 22:931-946. doi:10.1111/1539-6924.00262

21. IPCC (2001). Climate change 2001: the scientific basis. Intergovernmental Panel on Climate Change, pp 385-391. Available at http://www.grida.no/climate/ipcc_tar/

22. Hu XT, Zhu JX, Ding Q (2011) Environmental life-cycle comparisons of two polychlorinated biphenyl remediation technologies: incineration and base catalyzed decomposition. J Hazard Mater 191:258-268. doi:10.1016/j.jhazmat.2011.04.073

23. Zhu JX, Xie YJ, Zhang FS (2012) Life cycle analysis for PFOS decomposition in supercritical water. Procedia Environ Sci 16:304-310. doi:10.1016/j.proenv.2012.10.043 